J. Lake Sci. (湖泊科学), 2013, 25(3): 414-421

http: //www. jlakes.org. E-mail: jlakes@niglas.ac.cn

(C) 2013 by Journal of Lake Sciences

\title{
过量 $\mathrm{Zn}^{2+}$ 对水花生 ( Alternanthera philoxeroides) 愈伤组织氧化 胁迫及多胺代谢的影响
}

\author{
汪鹏合, 施国新林, 姜 岩, 陈霖, 乔绪强, 徐君, 贾 荣, 田秀丽 \\ （南京师范大学生命科学学院,江苏省生物多样性与生物技术重点实验室,南京 210023）
}

\begin{abstract}
摘 要: 以植物组织培养技术培养的水花生愈伤组织为实验材料, 研究了过量 $\mathrm{Zn}^{2+}(0 、 0.2 、 0.4 、 0.8 、 1.6 \mathrm{mmol} / \mathrm{L})$ 对水 花生愈伤组织的氧化损伤及多胺代谢的影响. 结果表明, 随着 $\mathrm{Zn}^{2+}$ 浓度增加 : (1) 水花生愈伤组织的超氧阴离子产生速 率和硫代巴比妥酸反应物水平显著提升, 超氧化物歧化酶活性明显抑制, 叶绿素 a 含量显著降低; (2) 精氨酸脱羧酶和鸟 氨酸脱羧酶活性均升高, 多胺氧化酶和二胺氧化酶活性在低 $\mathrm{Zn}^{2+}$ 浓度处升高、高 $\mathrm{Zn}^{2+}$ 浓度处降低; (3) 总腐胺和总精胺 含量在低 $\mathrm{Zn}^{2+}$ 浓度处降低、高 $\mathrm{Zn}^{2+}$ 浓度处增加, 总亚精胺含量仅在 $\mathrm{Zn}^{2+}$ 浓度为 $1.6 \mathrm{mmol} / \mathrm{L}$ 时有显著增加; (4) 游离态 腐胺含量变化与总腐胺含量相似, 游离态亚精胺含量在高 $\mathrm{Zn}^{2+}$ 浓度时下降, 游离态精胺含量仅在 $\mathrm{Zn}^{2+}$ 浓度为 $0.2 \mathrm{mmol} / \mathrm{L}$ 时略有下降; (5) 结合态腐胺和精胺在 $\mathrm{Zn}^{2+}$ 浓度为 $0.2 \mathrm{mmol} / \mathrm{L}$ 时下降而在其余处理组中上升, 结合态亚精 胺含量各处理组中均增加; (6) 束缚态腐胺和亚精胺含量均下降. 可见, 过量 $\mathrm{Zn}^{2+}$ 胁迫导致水花生愈伤组织氧化性损伤, 并扰乱其多胺代谢平衡; 束缚态多胺向结合态多胺的转化是过量 $\mathrm{Zn}^{2+}$ 胁迫下水花生愈伤组织多胺代谢变化的一个显著 特征; 以结合态为代表的内源性多胺积累对水花生愈伤组织抵抗过量 $\mathrm{Zn}^{2+}$ 胁迫具有重要意义.
\end{abstract}

关键词: 水花生愈伤组织;氧化胁迫;多胺代谢; $\mathrm{Zn}^{2+}$

\section{Oxidative stress and changes in polyamines metabolism caused by excessive zinc in Al- ternanthera philoxeroides callus}

WANG Penghe, SHI Guoxin, JIANG Yan, CHEN Lin, QIAO Xuqiang, XU Jun, JIA Rong \& TIAN Xiuli (Jiangsu Key Laboratory of Biodiversity and Biotechnology, College of Life Science, Nanjing Normal University, Nanjing 210023, P. R. China)

Abstract: Alternanthera philoxeroides callus, cultured by tissue culture technique, was exposed to gradient concentration of $\mathrm{Zn}^{2+}$ $(0,0.2,0.4,0.8,1.6 \mathrm{mmol} / \mathrm{L})$ for $7 \mathrm{~d}$. Oxidative stress and changes in polyamines metabolism caused by excessive zinc were investigated. Results showed that, with the increasing of $\mathrm{Zn}^{2+}$ concentration: (1) the rate of $\mathrm{O}_{2}^{-}$. generation and thiobarbituric acid reacting substances content was enhanced remarkably, and the superoxide dismutase activity and the chlorophyll-a content were decreased singnificantly; (2) the activities of arginine decarboxylase and ornithine decarboxylase were increased gradually, while the activities of polyamine oxidases and diamine oxidases were increased at first and then decreased at higher concentrations; (3) the contents of total-putrescine and total-spermine was decreased at first and then increased, and the contents of total-spermidine only showed an remarkable increase at $1.6 \mathrm{mmol} / \mathrm{L} \mathrm{Zn}^{2+}$ treatment; (4) the free-putrescine content changed in a similar pattern as its total counterpart, the free-spermidine content showed a decline at $0.8 \mathrm{mmol} / \mathrm{L}$ and $1.6 \mathrm{mmol} / \mathrm{L} \mathrm{Zn}^{2+}$ treatment, the free-spermine content had little fluctuation except a decrease at $0.2 \mathrm{mmol} / \mathrm{L} \mathrm{Zn}^{2+}$; (5) the contents of perchloric acid soluble conjugated (PS-conjugated) putrescine and spermine declined at $0.2 \mathrm{mmol} / \mathrm{L} \mathrm{Zn}^{2+}$ treatment and then increased, the PS-conjugated spermine content continued to rise; and (6) the contents of perchloric acid insoluble bound (PIS-bound) putrescine and spermine were descended gradually. The data presented here showed that excessive $\mathrm{Zn}^{2+}$ treatment can cause evident oxidative stress and alter the polyamines metabolism balance. The conversion from PIS-bound polyamines to PS-conjugated polyamines was one of the key characters of polyamines metabolism alternation in $A$. philoxeroides callus under excessive $\mathrm{Zn}^{2+}$ treatment. The accumulation in the total polyamines characterized by the PS-conjugated polyamines played an important role in A. philoxeroides cal-

* 江苏高校优势学科建设工程项目 (164320H106) 资助. 2012-07-10 收稿;2012-08-20 收修改稿. 汪鹏合, 男, 1989 年生, 硕士研究生; E-mail : wangpenghe0610222@ 163. com.

** 通信作者;E-mail:gxshi@ njnu. edu.cn. 
lus in response to excessive $\mathrm{Zn}^{2+}$ treatment.

Keywords: Alternanthera philoxeroides callus; oxidative stress; polyamines metabolism; $\mathrm{Zn}^{2+}$

作为仅有的一种参与所有六大类酶构成的金属,锌在多种植物代谢途径中扮演着重要角色 ${ }^{[1]}$. 但在高 浓度时, 它又能引起毒害作用. 过量的锌会影响植物根、叶等器官, 进而影响植株的高度和生物量 ${ }^{[2-5]}$. 近年 来,由锌造成的环境污染越来越严重,在水环境中也不例外 ${ }^{[6-7]}$. 然而, 大多数已有的研究都集中于天蓝遏蓝 菜、黄芥子、骆驼蹄瓣、毛蕊花和柳树等陆生植物,针对水生植物的相关研究很少 ${ }^{[8-12]}$.

氧化胁迫是重金属对植物造成毒害的重要途径之一,超氧阴离子 $\left(\mathrm{O}_{2}^{-} \cdot\right)$ 产生速率、硫代巴比妥酸反应物 (TBARs) 水平、超氧化物歧化酶 (SOD) 活性以及光合色素含量的变化常常被作为衡量氧化胁迫程度的指 标 ${ }^{[13-14]}$. 另外, 多胺 $(\mathrm{PAs})$ 作为一种含氮次生代谢产物, 其在植物抵御氧化胁迫中的作用也已引起广泛关 注 ${ }^{[15-16]}$. 游离态多胺被认为可以在重金属诱导的氧化胁迫中充当膜质过氧化抑制剂和羟基自由基的清除 剂 ${ }^{[17-18]}$. Papadalis 等指出多胺可能通过抑制 NADPH 氧化而淬灭活性氧, 保护大分子 ${ }^{[19]}$. Yi 等的相关研究表 明 Spd、Spm 能够通过减少镉吸收和避免 $\mathrm{H}_{2} \mathrm{O}_{2}$ 的产生来减少镉对水稻叶的氧化损伤 ${ }^{[20]}$. 而且, 关于逆境胁迫 下植物愈伤组织中多胺的保护性作用也有一些报道 ${ }^{[21-23]}$. 不过, 哪些种类或形式的多胺在植物抵御重金属 诱导的氧化胁迫中起主要作用在各研究中却不尽相同, 游离态腐胺在这一过程中所扮演的准确角色也仍然 有争议 ${ }^{[15]}$.

本实验以植物组织培养技术培养的水花生 (Alternanthera philoxeroides (Mart.) Griseb) 愈伤组织为实验 材料. 该材料作为一种生长在完全人工条件下的均一有机体, 具有重复性强,数据可靠、准确等特点. 本实验 主要研究过量锌对水花生愈伤组织的氧化胁迫和多胺代谢的影响, 旨在揭示过量锌对水花生愈伤组织的影 响以及可能的抗逆性机制,并为进一步的研究提供理论依据.

\section{1 材料和方法}

\section{1 供试材料}

水花生又名空心莲子草、喜旱莲子草, 为苋科莲子草属多年生宿根草本植物. 本实验以水花生茎段诱导 产生的愈伤组织为研究对象.

\section{2 实验方法}

1.2.1 愈伤组织的培养 选取幼嫩的水花生茎段作为外植体, 用肥皇水浸泡后自来水流动冲洗干净, 然后依 次经过 $70 \%$ 酒精 $30 \mathrm{~s} 、 5 \%$ 次氯酸钠 $10 \mathrm{~min}$ 和 $0.1 \%$ 升录 $10 \mathrm{~min}$ 消毒, 无菌水清洗 6 次后接种于添加 6-BA $(6.0 \mathrm{mg} / \mathrm{L})+\mathrm{NAA}(0.4 \mathrm{mg} / \mathrm{L})$ 的 $1 / 2 \mathrm{MS}$ 培养基 $(\mathrm{pH}$ 为 $5.8 \sim 6.0)$ 中, 放人 Forma 3744 控光、控温、控湿全 封闭大型培养箱 (英国产) 中培养愈伤组织. 培养箱温度为 $25^{\circ} \mathrm{C}$, 每天光照 $16 \mathrm{~h}$, 光照强度为 $1200 \sim 1500 \mathrm{~lx}^{[24]}$.

1.2.2 水花生愈伤组织过量锌胁迫处理 选取生长状况一致的致密型水花生愈伤组织置于含 $0 、 0.2 、 0.4$ 、 0.8 和 $1.6 \mathrm{mmol} / \mathrm{L} \mathrm{ZnSO}_{4}$ ( 以纯 $\mathrm{Zn}^{2+}$ 计) 的无菌液体培养基中, 培养条件同培养愈伤组织时一致. 第 $7 \mathrm{~d}$ 取相 同部位的愈伤组织块, 去离子水洗净、指干,进行各指标测定. 实验重复 3 次.

1.2.3 $\mathrm{O}_{2}^{-}$. 产生速率、TBARs 含量、SOD 活性及 $\mathrm{Chl}$. a 含量的测定超氧阴离子 $\left(\mathrm{O}_{2}^{-}\right.$. $)$含量用羟胺氧化反应 法测定 ${ }^{[25]}$; 硫代巴比妥酸反应物 (TBARs) 含量采用 Hodges 等的方法测定 ${ }^{[26]}$; 超氧化物歧化酶 ( SOD ) 活性 测定采用 NBT 光化还原法 ${ }^{[27]}$; 叶绿素 $\mathrm{a}\left(\right.$ Chl. a) 含量按 Liu 等的方法测定 ${ }^{[28]}$.

1.2 .4 多胺含量及其代谢酶活性的测定 精氨酸脱酸酶 ( ADC) 和鸟氨酸脱羧酶 $(\mathrm{ODC})$ 的活性根据赵福庚 等的方法测定 ${ }^{[29]}$; 多胺氧化酶 (PAO) 和二胺氧化酶 (DAO) 的活性测定参照汪天等的方法 ${ }^{\left[{ }^{[30]}\right.}$; 多胺 $(P A s)$ 含 量测定采用 Aziz 等的方法进行 ${ }^{[31]}$.

\section{3 统计分析}

实验结果为 3 次实验的平均值 \pm 标准差, 实验原始数据的处理和制图采用 Excel 2007 和 SPSS 17.0 软 件完成. 将各生理指标与 $\mathrm{Zn}^{2+}$ 处理浓度作相关性分析, 其中 $P \geqslant 0.05$ 为无显著相关; $P<0.05$ 为显著相关; $P<0.01$ 为极显著相关. 将各生理指标不同处理组之间进行差异显著性分析, 图中不同小写字母表示数值之 间差异显著 $(P<0.05)$, 相同字母表示数值之间差异不显著. 


\section{2 结果与分析}

\section{1 过量 $\mathrm{Zn}^{2+}$ 对水花生愈伤组织的氧化性损伤}

随着 $\mathrm{Zn}^{2+}$ 浓度的增加, $\mathrm{O}_{2}^{-}$. 产生速率逐渐提高, 在 $1.6 \mathrm{mmol} / \mathrm{L}$ 时是对照的 2.0 倍 (图 $1 \mathrm{~A}$ ); 差异分析表 明,其变化与 $\mathrm{Zn}^{2+}$ 处理浓度达到极显著正相关 $(r=0.9379, P<0.01)$. 同时, TBARs 的含量也出现不同程度 的升高, $\mathrm{Zn}^{2+}$ 浓度为 $0.2 \mathrm{mmol} / \mathrm{L}$ 时含量最高, 大小是对照的 1.92 倍, 而最小幅度的增加发生在 $1.6 \mathrm{mmol} / \mathrm{L}$ $\mathrm{Zn}^{2+}$ 时, 其大小是对照的 1.34 倍 (图 1B). 此外, 超氧化物歧化酶 (SOD) 活性和叶绿素 a ( Chl. a ) 含量随着 $\mathrm{Zn}^{2+}$ 浓度的增加而逐渐降低, 在 $1.6 \mathrm{mmol} / \mathrm{L}$ 时仅分别为对照的 7.97\% 和 43.4\% (图 1C、D). 差异分析表明, SOD 活性及 Chl. a 含量的变化与 $\mathrm{Zn}^{2+}$ 处理浓度分别达到极显著负相关和显著负相关 $\left(r_{\mathrm{SOD}}=-0.9846\right.$, $\left.P_{\mathrm{SOD}}<0.01 ; r_{\text {Chl. a }}=-0.8552, P_{\text {Chl. a }}<0.05\right)$.
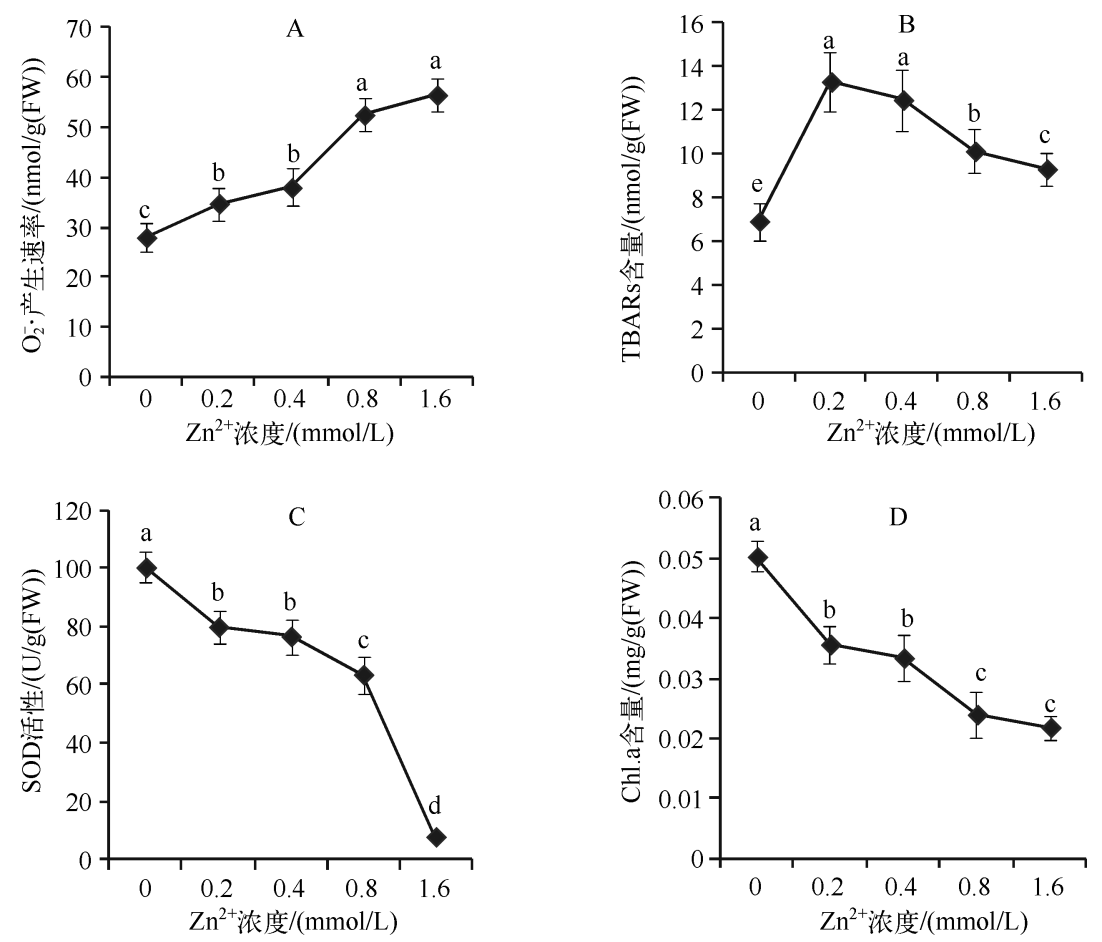

图 1 过量 $\mathrm{Zn}^{2+}$ 对水花生愈伤组织的氧化性胁迫的影响

Fig. 1 Effects of oxidative stress in A. philoxeroides callus after $7 \mathrm{~d}$ of excessive $\mathrm{Zn}^{2+}$ treatment

\section{2 过量 $\mathrm{Zn}^{2+}$ 对水花生愈伤组织中 ADC、ODC、PAO 和 DAO 活性的影响}

随着 $\mathrm{Zn}^{2+}$ 浓度的升高, 合成 Put 的两个关键酶— ADC 和 ODC 活性均逐步上升, 在 $1.6 \mathrm{mmol} / \mathrm{L}$ 时达到 最大值, 分别为 $175.01 \mathrm{U} / \mathrm{g}(\mathrm{FW})$ 和 $4.11 \mathrm{U} / \mathrm{g}(\mathrm{FW})$, 分别是对照组的 3.60 和 1.22 倍 (图 $2 \mathrm{~A}$ 和图 $2 \mathrm{~B}$ ). 统计 分析表明,除 $0.2 \mathrm{mmol} / \mathrm{L} \mathrm{Zn}^{2+}$ 处理组外, 其余处理组中 ADC 和 ODC 活性的变化与对照组相比均达显著差 异水平 $(P<0.05)$. $\mathrm{ADC}$ 和 $\mathrm{ODC}$ 活性的变化与 $\mathrm{Zn}^{2+}$ 浓度之间均呈极显著正相关 $\left(r_{\mathrm{ADC}}=0.9746, r_{\mathrm{ODC}}=\right.$ $0.9658 ; P<0.01)$.

$\mathrm{PAO}$ 和 DAO 活性则呈现出在低 $\mathrm{Zn}^{2+}$ 浓度胁迫时升高、高 $\mathrm{Zn}^{2+}$ 浓度胁迫时下降的趋势. PAO 活性在 $\mathrm{Zn}^{2+}$ 浓度为 $0.2 \mathrm{mmol} / \mathrm{L}$ 及 $0.4 \mathrm{mmol} / \mathrm{L}$ 胁迫时均达到最大值 $2.5 \mathrm{U} / \mathrm{g}(\mathrm{FW})$, 为对照的 2.00 倍, 而在更高 $\mathrm{Zn}^{2+}$ 浓度胁迫时下降到对照水平 (图 2C) ; DAO 则在 $\mathrm{Zn}^{2+}$ 浓度为 $0.8 \mathrm{mmol} / \mathrm{L}$ 时胁迫达到最大值 $3.4 \mathrm{U} / \mathrm{g}(\mathrm{FW}$ ), 为 对照的 2.80 倍, 而在 $\mathrm{Zn}^{2+}$ 浓度为 $1.6 \mathrm{mmol} / \mathrm{L}$ 时略有下降, 但仍比对照组高, 为对照的 1.67 倍 (图 2D). 统计 分析表明, 各 $\mathrm{Zn}^{2+}$ 处理组中 PAO 和 DAO 活性的变化与对照组相比均达显著差异水平 $(P<0.05)$. 

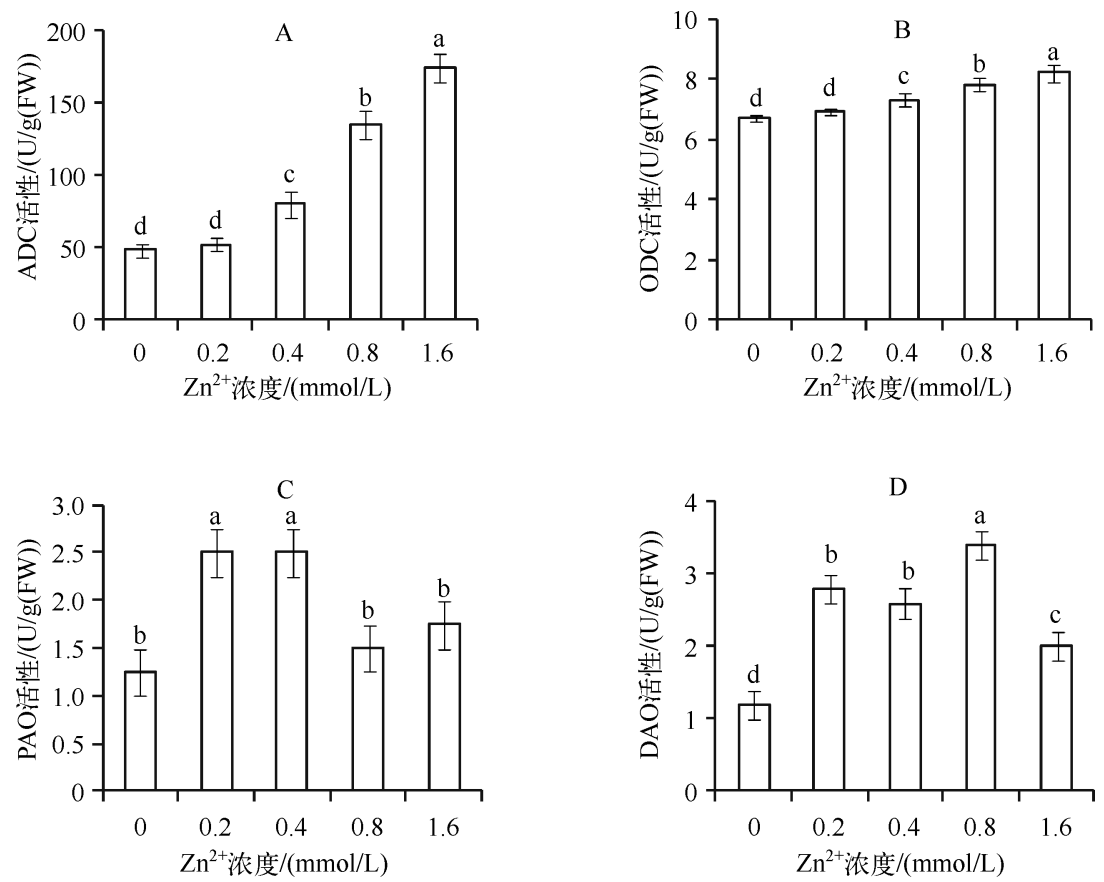

图 2 过量 $\mathrm{Zn}^{2+}$ 对水花生愈伤组织中 $\operatorname{ADC}(\mathrm{A}) 、 \mathrm{ODC}(\mathrm{B}) 、 \mathrm{PAO}(\mathrm{C})$ 和 $\mathrm{DAO}(\mathrm{D})$ 活性的影响

Fig. 2 Activities of arginine decarboxylase (ADC) (A), ornithine decarboxylase ( ODC) (B), polyamine oxidase $(\mathrm{PAO})(\mathrm{C})$ and diamine oxidase (DAO) (D) in A. philoxeroides callus after $7 \mathrm{~d}$ of excessive $\mathrm{Zn}^{2+}$ treatment

\section{3 过量 $\mathrm{Zn}^{2+}$ 对水花生愈伤组织中 PAs 含量的影响}

2.3.1 总多胺 (t-PAs) 含量的变化 随着 $\mathrm{Zn}^{2+}$ 浓度的升高, 水花生愈伤组织中总腐胺 (Put) 与总精胺 ( Spm) 含量呈现出在低 $\mathrm{Zn}^{2+}$ 浓度时降低、高 $\mathrm{Zn}^{2+}$ 浓度时增加的趋势. 在 $\mathrm{Zn}^{2+}$ 浓度为 $0.2 \mathrm{mmol} / \mathrm{L}$ 时, 两者含量最 低, 分别为对照的 $76.8 \%$ 和 $61.4 \%$; 而后逐渐增加, 并在 $\mathrm{Zn}^{2+}$ 浓度为 $1.6 \mathrm{mmol} / \mathrm{L}$ 时达到最大值, 分别为对照 的 1.42 倍和 1.83 倍. 而总亚精胺 ( $\mathrm{Spd}$ ) 含量则在较低 $\mathrm{Zn}^{2+}$ 浓度处理时无显著变化, 至 $\mathrm{Zn}^{2+}$ 浓度达 $1.6 \mathrm{mmol} / \mathrm{L}$ 时才显著增加, 为对照组的 1.34 倍 (图 $3 \mathrm{~A}$ ).

2.3 .2 游离态多胺 (f-PAs) 含量的变化 随着 $\mathrm{Zn}^{2+}$ 浓度的升高, 水花生愈伤组织中游离态 Put ( f-Put) 含量变 化呈现出与总 Put 含量相似的变化趋势, 在 $\mathrm{Zn}^{2+}$ 浓度为 $0.2 \mathrm{mmol} / \mathrm{L}$ 时含量最低, 为对照的 $75.0 \%$; 在 $\mathrm{Zn}^{2+}$ 浓度为 $1.6 \mathrm{mmol} / \mathrm{L}$ 时含量最高, 为对照的 2.36 倍. 而游离态 $\mathrm{Spd}\left(\mathrm{f}-\mathrm{Spd}\right.$ ) 则呈现出不同的趋势, 在 $\mathrm{Zn}^{2+}$ 浓度 为 0.2 和 $0.4 \mathrm{mmol} / \mathrm{L}$ 时, 其含量和对照相比无显著差异, 在 $\mathrm{Zn}^{2+}$ 浓度达到 $0.8 \mathrm{mmol} / \mathrm{L}$ 时, 其含量开始降低, 最低值在 $\mathrm{Zn}^{2+}$ 浓度为 $1.6 \mathrm{mmol} / \mathrm{L}$ 处, 为对照的 $55.5 \%$. 游离态 $\mathrm{Spm}(\mathrm{f}-\mathrm{Spm})$ 仅在 $\mathrm{Zn}^{2+}$ 为 $0.2 \mathrm{mmol} / \mathrm{L}$ 时略有 下降(图 3B).

2.3.3 结合态多胺 (PS-PAs) 含量的变化 随着 $\mathrm{Zn}^{2+}$ 浓度的升高, 水花生愈伤组织中结合态 Put (PS-Put) 和 结合态 Spm (PS-Spm) 的含量也呈现出与总 Put 及总 Spm 含量变化相似的趋势. 它们在 $\mathrm{Zn}^{2+}$ 浓度为 $0.2 \mathrm{mmol} / \mathrm{L}$ 时降低, 分别为对照的 $80.7 \%$ 和 $59.0 \%$, 而在其余各处理组中升高, 并于 $\mathrm{Zn}^{2+}$ 浓度为 $1.6 \mathrm{mmol} / \mathrm{L}$ 时达到最大, 含量分别为对照的 1.98 倍和 2.04 倍. 结合态 $\mathrm{Spd}$ (PS-Spd) 含量则随着 $\mathrm{Zn}^{2+}$ 浓度的升高逐步上 升, 在 $\mathrm{Zn}^{2+}$ 浓度为 $1.6 \mathrm{mmol} / \mathrm{L}$ 时达到最大值, 为对照的 2.03 倍 (图 3C).

2.3.4 束缚态多胺 (PIS-PAs) 含量的变化 随着 $\mathrm{Zn}^{2+}$ 浓度的升高, 水花生愈伤组织中束缚态 Put (PIS-Put) 和 束缚态 $\operatorname{Spd}\left(\mathrm{PIS}-\mathrm{Spd}\right.$ ) 含量降低, 最小值出现在 $\mathrm{Zn}^{2+}$ 浓度为 $0.8 \mathrm{mmol} / \mathrm{L}$ 时, 分别为对照的 $52.7 \%$ 和 $24.8 \%$ ( 图 3D). 束缚态 Spm 含量没有被检测到. 

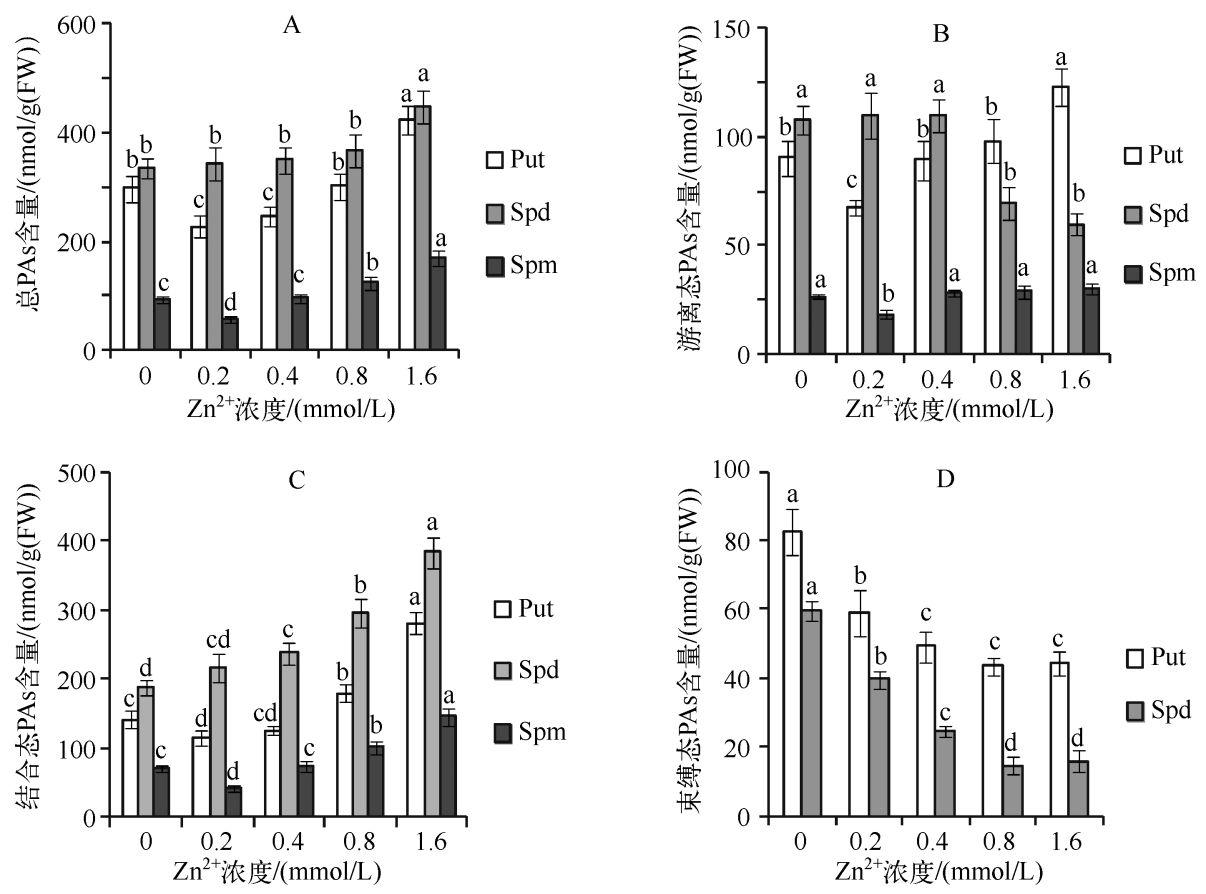

图 3 过量 $\mathrm{Zn}^{2+}$ 对水花生愈伤组织中总体 $(\mathrm{A})$ 、游离态 $(\mathrm{B})$ 、结合态 $(\mathrm{C})$ 和束缚态 $(\mathrm{D})$ 多胺 (Put,Spd,Spm) 含量的影响

Fig. 3 Changes of the contents of PAs (Put, Spd, Spm) in total (A), free (B), PS-conjugated (C) and PIS-conjugated (D) forms in A. philoxeroides callus after $7 \mathrm{~d}$ of excessive $\mathrm{Zn}^{2+}$ treatment

\section{3 讨论}

氧化胁迫是重金属毒害的一个普遍特征 ${ }^{[32]}$. $\mathrm{O}_{2}^{-}$. 等自由基 (ROS) 的积累与生物膜的损伤密切相关, 而 后者的程度常通过 TBARs 的水平来反映 ${ }^{[13]}$. 本实验中, $\mathrm{Zn}^{2+}$ 浓度与 $\mathrm{O}_{2}^{-}$. 产生速率呈显著的正相关关系. 同 时,在所有处理组中 TBARs 的水平均高于对照. 这表明过量 $\mathrm{Zn}^{2+}$ 胁迫导致了 ROS 的产生以及膜脂过氧化的 增强. 其具体机制可能涉及重金属胁迫对电子传递的影响 ${ }^{[33]}$. SOD 可以催化 $\mathrm{O}_{2}^{-}$. 转变为 $\mathrm{H}_{2} \mathrm{O}_{2}$ 和 $\mathrm{O}_{2}$. 有研究 指出其活性大小依赖于植物细胞所受到的氧化胁迫程度 ${ }^{[34]}$. 而本实验的结果却与之相反, 随着 $\mathrm{Zn}^{2+}$ 浓度的 提高, $\mathrm{O}_{2}^{-}$. 产生速率的增加, $\mathrm{SOD}$ 的活性却逐步降低. 可见, 在过量 $\mathrm{Zn}^{2+}$ 处理下水花生愈伤组织中 SOD 活性 随着氧化胁迫程度增加而降低, 这可能与底物过量所导致的酶活性抑制有关 ${ }^{[35]}$. 在植物体中, 重金属诱导的 氧化胁迫下光合色素的损伤也常见于报道, Macfarlane 等在研究中曾把光合色素作为锌胁迫的一个敏感指 标 ${ }^{[36]}$. Chl. a 作为主要的光合色素, 在诸如铜、铅、镉等重金属胁迫研究中常呈下降趋势 ${ }^{[36-37]}$. 当前的实验结 果表明过量的锌也能降低 Chl. a 的含量,氧化胁迫下生物膜的损伤尤其是膜通透性的改变被认为是一个重 要原因 ${ }^{[36]}$, 本实验中 TBARs 含量的增加也证实了这一点.

多胺主要包括 Put、Spd 和 Spm, 在植物体内以游离态 ( free-) 、高氯酸可溶性结合态 (PS-) 和高氯酸不溶 性结合态 (PIS-) 形式存在, 其含量及代谢平衡的维持在植物抵御氧化胁迫中起重要作用 ${ }^{[15-16,38]}$. 参与多胺代 谢平衡调节的主要有两组酶类: ADC 和 ODC 分别以精氨酸和鸟氨酸为底物合成 $\mathrm{Put}^{[39]}$, 而 Put 可以作为 $\mathrm{Spd}$ 和 Spm 的直接底物; PAO 和 DAO 分别以 Spm 和 Put 为最适底物催化 PAs 的氧化分解 ${ }^{[4041]}$. 本研究中, ADC 和 ODC 的活性随着 $\mathrm{Zn}^{2+}$ 浓度的增加逐渐增强, 而 PAO 和 DAO 的活性表现出低 $\mathrm{Zn}^{2+}$ 浓度下升高、高 $\mathrm{Zn}^{2+}$ 浓 度下降低的趋势. 这表明过量 $\mathrm{Zn}^{2+}$ 能够不同程度地影响两组关键酶的活性,进而改变多胺代谢平衡以适应 重金属胁迫,这也与其他研究者观察到的镉、铜等重金属胁迫具有类似效应的结果相一致 ${ }^{[38,4243]}$. 早期的研 究揭示, $\mathrm{ADC}$ 和 ODC 的活性主要分别存在于叶绿体和胞质溶胶中,而 PAO 和 DAO 的活性位于细胞壁 ${ }^{[44-46]}$, 
这可能是两组酶对 $\mathrm{Zn}^{2+}$ 浓度敏感性不同的原因. 两组酶在 $\mathrm{Zn}^{2+}$ 胁迫下不同程度的激活, 导致了总体多胺含 量在较低 $\mathrm{Zn}^{2+}$ 浓度时降低而后随着 $\mathrm{Zn}^{2+}$ 浓度的增加而增加. 在较低 $\mathrm{Zn}^{2+}$ 浓度时总 Put 和总 Spm 含量的减 少可以归因于升高的 $\mathrm{PAO}$ 与 DAO 活性以及较低的 ADC 与 ODC 活性, 正是这样的激活程度差异导致了 PAs 被大量氧化分解而不能得到及时补充; 在较高 $\mathrm{Zn}^{2+}$ 浓度时整体多胺含量的上升则是由于 ADC 和 ODC 活性 的提高以及 PAO 和 DAO 活性的抑制.

和以往相关领域的研究相比,本研究在多胺含量变化平衡方面呈现出一些不同的特点. 先前的研究经 常报道同一种类不同形式的 PAs 在重金属胁迫下的变化遵循相似的趋势 ${ }^{[38,47-48]}$, 而这种情况在本研究中却 没有出现. 本研究表明,同一种类但不同形式的 PAs 对过量 $\mathrm{Zn}^{2+}$ 胁迫呈现出不同的响应特征, 而这些不同的 变化具有不同的意义.

早先的一项研究认为外施的 Put 会抑制 SOD 的活性但是又能通过降低 $\mathrm{O}_{2}^{-}$. 的积累而提高植物原生质 体存活率 ${ }^{[49]}$; 而另一些研究者却指出过量的 f-Put 对植物具有毒害作用 ${ }^{[50-51]}$. 在本试验中, 随着 $\mathrm{Zn}^{2+}$ 浓度的 增加,f-Put 或总 Put 含量的变化趋势均没有被发现与 $\mathrm{O}_{2}^{-}$. 产生速率、SOD 活性以及 Chl. a 含量等胁迫指标的 变化趋势具有平行性. 所以在过量锌胁迫下的水花生愈伤组织中 f-Put 只是作为一个普通的胁迫响应参与者 而非保护者. 另一方面, 本文观察到在高浓度 $\mathrm{Zn}^{2+}$ 条件下 f-Spd 含量降低, 较高水平的 Spd 含量更有利于生 物膜的稳定, f-Spd 水平的下降被认为会降低植物抗逆性 ${ }^{[52]}$, 这也与其它一些已经发表的研究报告如镍处理 下的水鳖、盐胁迫下的荷花以及镉毒害下的竹叶眼子菜等相一致 ${ }^{[38,47,53]}$.

PS-PAs 是水花生愈伤组织中各不同形态多胺含量最高的, 其含量的变化主导了总体多胺水平的变化. 这暗示了 PS-PAs 在水花生愈伤组织对过量 $\mathrm{Zn}^{2+}$ 胁迫的响应中起主要作用. 本实验中 PS-PAs 水平随着 $\mathrm{Zn}^{2+}$ 浓度增加而持续升高, 这与田秀丽等的研究结果一致 ${ }^{[54]}$. PS-PAs 可以维持质膜、线粒体膜等生物膜的完整 性 $^{[55]}$, 其含量的增加对植物细胞具有一定的保护作用 ${ }^{[56]}$.

PIS-PAs 积极参与盐胁迫、氧化胁迫和重金属胁迫等逆境条件下植物适应性反应的现象也已有所报

道 ${ }^{[47,57-58]}$. Yang 等在对竹叶眼子菜的研究时认为在逆境条件下 PIS-PAs 可以充当 f-PAs 的来源 ${ }^{[38]}$. 一个类似 的由 PIS-PAs 向 PS-PAs 转化的现象在铜处理的胡萝卜中也被观察到 ${ }^{[59]}$. 本实验中 PIS-PAs 水平并没有和 PS-PAs 一样随着胁迫的增强而升高, 反而呈现出下降趋势. 这可能是 PIS-PAs 向 PS-PAs 发生转化, 在一定程 度上导致了 PS-PAs 含量在 $\mathrm{Zn}^{2+}$ 胁迫下的增加,进而间接地提升了水花生愈伤组织的抗逆性.

综上所述,过量锌导致水花生愈伤组织产生了一系列的毒害症状. 其对细胞造成了氧化性胁迫并改变 了多胺代谢的平衡. 束缚态多胺向结合态多胺的转化是过量 $\mathrm{Zn}^{2+}$ 胁迫下水花生愈伤组织多胺代谢变化的一 个显著特征; 结合态多胺的积累对水花生愈伤组织抵抗过量 $\mathrm{Zn}^{2+}$ 胁迫具有重要意义; 游离态多胺同样积极 参与水花生愈伤组织对过量 $\mathrm{Zn}^{2+}$ 胁迫的响应. 两组多胺代谢关键酶 ADC/ODC 和 PAO/DAO 在这一过程中 扮演着重要的调节角色. 更详细机制有待进一步探究.

\section{4 参考文献}

[ 1 ] Broadley MR, White PJ, Hammond JP et al. Zinc in plants. New Phytol, 2007,173 : 677-702.

[ 2 ] Shetty KG, Hetrick BAD, Schwab AP. Effects of mycorrhizae and fertilizer amendments on zinc tolerance of plants. Environ Pollut, 1995 ,88 : 307-314.

[ 3 ] Ozturk L, Karanlik S, Ozkutlu F et al. Shoot biomass and zinc/cadmium uptake for hyperaccumulator and non-accumulator Thlaspi species in response to growth on a zinc deficient calcareous soil. Plant Sci, 2003,164: 1095-1101.

[ 4 ] Castiglione S, Franchin C, Fossati T et al. High zinc concentrations reduce rooting capacity and alter metallothionein gene expression in white poplar (Populus alba L. cv. Villafranca). Chemosphere, 2007,67 : 1117-1126.

[ 5 ] Todeschini V, Lingua G, D’Agostino G et al. Effects of high zinc concentration on poplar leaves: A morphological and biochemical study. Environ Exp Bot, 2011,71: 50-56.

[6] Akcay H, Oguz A, Karapire C. Study of heavy metal pollution and speciation in Buyak Menderes and Gediz river sediments. Water Res, 2003, 37: 813-822.

[ 7 ] Kar D, Sur P, Mandal SK, Saha T et al. Assessment of heavy metal pollution in surface water. Int J Environ Sci Tech, 2008, 5 : 119-124.

[ 8 ] Salt DE, Prince RC, Baker AJM et al. Zinc ligands in the metal hyperaccumulator Thlaspi caerulescens as determined 
using X-ray absorption spectroscopy. Environ Sci Technol, 1999,33 : 713-717.

[ 9 ] Prasad KVSK, Saradhi PP, Sharmila P. Concerted action of antioxidant enzymes and curtailed growth under zinc toxicity in Brassica junca. Environ Exp Bot, 1999, 42 : 1-10.

[10] Lefèvre I, Correal E, Lutts S. Impact of cadmium and zinc on growth and water status of Zygophyllum fabago in two contrasting metallicolous populations from SE Spain: comparison at whole plant and tissue level. Plant Biology, 2009 ,12 : $883-894$.

[11] Morina F, Jovanovicb L, Mojovicc M et al. Zinc-induced oxidative stress in Verbascum thapsus is caused by an accumulation of reactive oxygen species and quinhydrone in the cell wall. Physiol Plantarum, 2010 ,140 : 209-224.

[12] Landberg T, Jensén P, Greger M. Strategies of cadmium and zinc resistance in willow by regulation of net accumulation. Biol Plantrum, 2011,55: 133-140.

[13] Rellán-ÁNarez R, Ortega-Villasante C, Álvarez-Fernández A et al. Stress responses of Zea mays to cadmium and mercury. Plant Soil, 2006, 279: 41-50.

[14] Macfarlane GR, Burchett MD. Photosynthetic pigments and peroxidase activity as indicators of heavy metal stress in the grey mangrove, Avicennia marina (forsk.) Vierh. Mar Pollut Bull, 2001,42 : 233-240.

[15] Alcázar R, Marco F, Cuevas JC et al. Involvement of polyamines in plant response to abiotic stress. Biotechnol Lett, 2006,28 : $1867-1876$.

[16] Groppa MD, Benavides MP. Polyamines and abiotic stress: recent advances. Amino Acids, 2008,34 : $35-45$.

[17] Metha SK, Gaur JP. Heavy-mecal-induced proline accumulation and its role in ameliorating metal toxicity in Chlorella vulgaris. New Phytol, 1999,143 : 253-259.

[18] Das KC, Misra HP. Hydroxyl radical scavenging and singlet oxygen quenching properties of polyamines. Mol Cell Biochem, 2004, 262 : 127-133.

[19] Papadalis AK, Roubelakis-Angelakis KA. Polyamines inhibit NADPH oxidase-mediated superoxides generation and putrescine prevents programed cell death induced by polyamine oxidase-generated hydrogen peroxide. Planta, 2005, 220 : 826-837.

[20] Yi TH, Ching HK. Cadmium-induced oxidative damage in rice leaves is reduced by polyamines. Plant Soil, 2007,291 :27-31.

[21] Alvarez I, Tomaro ML, Benavides MP. Changes in polyamines, proline and ethylene in sunflower calluses treated with NaCl. Plant Cell Tiss Organ Cult, 2003,74: 51-59.

[22] Tang W, Newton RJ. Increase of polyphenol oxidase and decrease of polyamines correlate with tissue browning in Virginia pine (Pinus virginiana Mill. ). Plant Sci, 2004, 167: 621-628.

[23] Tang W, Newton RJ. Polyamines reduce salt-induced oxidative damage by increasing the activities of antioxidant enzymes and decreasing lipid peroxidation in Virginia pine. Plant Growth Regul, 2005,46 : $31-43$.

[24] 袁 燕,生吉萍,王捍东等. 水花生愈伤组织的诱导及根的分化. 水生生物学报,2004,28(6):622-628.

[25] 王爱国,罗广华. 植物的超氧物自由基与差胺反应的定量关系. 植物生理学通讯, 1990,6 55-57.

[26] Hodges DM, Delong JM, Forney CF et al. Improving the thiobarbituric acid-reactive-substances assay for estimating lipid peroxidation in plant tissues containing anthocyanin and other interfering compounds. Planta, 1999, 207: 604-611.

[27] Giannopolitis CN, Ries SK. Superoxide dismutase in higher plants. Plant Physiol, 1977,59: 309-314.

[28] Liu XM, Wang JF, Yu RQ et al. Study on the changes of chlorophyll content and biomass of heterotrophic Chlorella vulgaris under different nitrogen concentrations. Plant Physiol, 1999,35 : 198-201.

[29] Zhao FG, Sun C, Liu YL et al. Relationship between polyamine metabolism in roots and salt tolerance of barley seedlings. Acta Bot Sin, 2003 ,45 : 295-300.

[30] 汪 天, 郭世荣, 刘 俊等. 多胺氧化酶检测方法的改进及其在低氧水培黄瓜根系中的应用. 植物生理学通讯, $2004,40: 358-360$.

[31] Aziz A, Larher F. Changes in polyamine titers associated with the proline response and osmotic adjustment of rape leaf discs submitted to osmotic stresses. Plant Sci, 1995,112 : 175-186.

[32] Mithöfer A, Schulze B, Boland W. Biotic and heavy metal stress response in plants: evidence for common signals. FEBS Letters, 2004,566: $1-5$.

[33] Atal N, Saradhi PP, Mohanty P. Inhibition of the chloroplast photochemical reactions by treatment of wheat seedlings with low concentration of cadmium: analysis of electron transport activities and changes in fluorescence yield. Plant Cell Physiol, 1991,32: 943-951.

[34 ] Chamseddine M, Wided BA, Guy H et al. Cadmium and copper induction of oxidative stress and antioxidative response in 
tomato (Solanum lycopersicon) leaves. Plant Growth Regul, 2009,57: 89-99.

[35] Demiral T, Túrkan I. Comparative lipid peroxidation, antioxidant defense systems and proline content in roots of two rice cultivars differing in salt tolerance. Environ Exp Bot, 2005,53 : 247-257.

[36] Macfarlane GR, Burchett MD. Phytosynthetic pigments and peroxidase activity as indicators of heavy metal stress in the grey mangrove, Avicewnnia marina (Forsk. ) Vierh. Mar pollut Bull, 2001, 42 : 233-240.

[37] Aravind P, Prasad MNV. Zinc protects chloroplasts and associated photochemical functions in cadmium exposed Ceratophyllum demersum L. a freshwater macrophyte. Plant Sci, 2004,166 : 1321-1327.

[38] Yang H, Shi G, Wang H et al. Involvement of polyamines in adaptation of Potamogeton crispus L. to cadmium stress. Aquat Toxicol, $2010, \mathbf{1 0 0}$ : 282-288.

[39] Martin-Tanguy J. Metabolism and function of polyamines in plants: recent development (new approaches) . Plant Growth Regul, 2001, 34 : 135-148.

[40] Walters DR. Polyamines and plant disease. Phytochemistry, 2003, 64: 97-107.

[41] Alcázar R, Altabella T, Marco F et al. Polyamines: molecules with regulatory functions in plant abiotic stress tolerance. Planta, 2010,231: 1237-1249.

[42] Groppa MD, Tomaro ML, Benavides MP. Polyamines and heavy metal stress : the antioxidant behavior of spermine in cadmium-and copper-treated wheat leaves. Biometals, 2007,20: 185-195.

[43] Xu X, Shi G, Ding C et al. Regulation of exogenous spermidine on the reactive oxygen species level and polyamine metabolism in Alternanthera philoxeroides (Mart. ) Griseb under copper stress. Plant Growth Regul, 2011, 63 : $251-258$.

[44] Slocum RD, Furey MG III. Electron-microscopic cytochemical localization of diamine and polyamine oxidases in pea and maize tissues. Planta, $1991, \mathbf{1 8 3}$ : 443-450.

[45] Borrell A, Culiañez-Macia FA, Altabella T et al. Arginine decarboxylase is localized in chloroplasts. Plant Physiol, 1995, 109: 771-776.

[46] Walker MA, Ellis BE, Chapple CCS et al. Subcellular localization of amines and activities of their biosynthetic enzymes in p-fluorophenylalanine resistant and wild-type tobacco cell cultures. Plant Physiol, $1987,85: 78-81$.

[47] Zhao J, Shi G, Yuan Q. Polyamines content and physiological and biochemical responses to ladder concentration of nickel stress in Hydrocharis dubia ( Bl. ) backer leaves. Biometals, 2008 ,21 : 665-674.

[48] Ding C, Shi G, Xu X et al. Effect of exogenous spermidine on polyamine metabolism in water hyacinth leaves under mercury stress. Plant Growth Regul, 2010,60: 61-67.

[49] Papadakis AK, Roubelakis-Angelakis KA. Polyamines inhibit NADPH oxidase-mediated superoxide generation and putrescine prevents programned cell death induced by polyamine oxidase-generated hydrogen peroxide. Planta, 2005, 220 : 826-837.

[50] Groppa MD, Tomaro ML, Benavides MP. Polyamines as protectors against cadmium or copper-induced oxidative damage in sunflower leaf discs. Plant Sci, 2001,161: $481-488$.

[51] Panicot M, Masgrau C, Borrell A et al. Effects of putrescine accumulation in tobacco transgenic plants with different expression levels of oat arginine decarboxylase. Physiol Plantrum, 2002 ,114: $281-287$.

[52] Bouchereau A, Aziz A, Larher F et al. Polyamines and environmental challenges: recent development. Plant Sci, 1999, 140 : $103-125$.

[53] Sanchez DH, Cuevas JC, Chiesa MA et al. Free spermidine and spermine content in Lotus glaber under long-term salt stress. Plant Sci, $2005, \mathbf{1 6 8}$ : 541-546.

[54] 田秀丽,施国新, 徐 君等. $\mathrm{Cr}^{6+}$ 胁迫对水花生愈伤组织多胺代谢的影响. 湖泊科学, 2011,23(6):955-960.

[55] 王红霞,胡金朝,施国新等. 外源多胺对铜胁迫下水鳖叶片多胺代谢、抗氧化系统和矿质营养元素的影响. 生态学 报,2010,30(10):2784-2792.

[56] Liu HP, Zhu ZX, Liu TX et al. Effect of osmotic stress on the kinds, forms and levels of polyamines in wheat coleoptiles. Journal of Plant Physiology and Molecular Biology, 2006,32(3) :293-299.

[57] Dondini L, Bonazzi S, Duca SD et al. Acclimation of chloroplast transglutaminase to high NaCl concentration in a polyamine-deficient variant strain of Dunaliella salina and in its wild type. J Plant Physiol, 2001,158 : 185-197.

[58] Roussos PA, Pontikis CA. Changes of free, soluble conjugated and bound polyamine titers of jojoba explants under sodium chloride salinity in vitro. J Plant Physiol, 2007 ,164:895-903.

[59] Górecka TK, Cvikrová M, Kowalska U et al. The impact of Cu treatment on phenolic and polyamine levels in plant material regenerated from embryos obtained in anther culture of carrot. Plant Physiol Bioch, 2007,45 : 54-61. 